\section{La Interdisciplinariedad un camino para la inserción de la Sustentabilidad en cursos de Diseño de Producto}

\author{
Ana Veronica Pazmino ${ }^{(1)}$
}

Resumen: Este escrito presenta una reflexión sobre la necesidad de la práctica interdisciplinar en el campo del diseño. Por medio de una investigación documental es presentada la complejidad de la sustentabilidad en el ámbito ambiental, humano, social, cultural y tecnológico, mostrando la importancia de la interdisciplinariedad como medio de integración con disciplinas que no hacen parte del plano curricular de los cursos de diseño. El trabajo enfatiza la necesidad de integrar disciplinas como biología, ecología, química, ingeniería ambiental, sociología, antropología con el campo del diseño de producto por medio del diálogo crítico y sólido promovido por una currícula que coloque estas disciplinas como optativas u obligatorias.

Palabras clave: Interdisciplinariedad - Diseño de producto - Sustentabilidad - Enseñanza del diseño - Disciplina

[Resúmenes en inglés y portugués en la página 91]

(1) Ana Verónica Pazmino es Licenciada en Diseño Industrial de la Universidad Federal de Río de Janeiro; Magíster en Ingeniería de Producción de la Universidad Federal de Santa Catarina; Doctora en Diseño por la PUC-Rio. Es profesora asociada de la Universidad Federal de Santa Catarina, UFSC. Tiene experiencia en el área de Diseño de Producto y Métodos de Proyecto, actuando principalmente en las siguientes líneas de investigación: Métodos de proyecto, diseño social, diseño ambiental y docencia del diseño. anaverpw@ gmail.com

\title{
Introducción
}

El conocimiento del diseñador de productos se organiza a partir de repertorios y paradigmas para crear ideas que se materializan en artefactos. El conocimiento, está profundamente relacionado a la cultura y a la sociedad recordando que estas se transforman, se desarrollan, progresan y vuelven. Para Tronca (2006, p. 65) el saber hoy tiene que ser repensado considerando la gran cantidad de información y saberes. 
Se sabe que el proceso de diseño es complejo ${ }^{1}$, puede parecer que complejidad supone confusión, desorden, falta de certeza, pero orden y desorden son procesos constitutivos de la complejidad y por tanto del proceso de diseño; de esa forma, el pensamiento del diseñador debe ser amplio, multidimensional, capaz de comprender la complejidad y construir un conocimiento que lleve en consideración esa misma amplitud.

Las relaciones de las fases de un proyecto que constituyen el proceso de diseño deben ser presididas por integraciones de métodos, conocimientos y teorías de diversas disciplinas. Así, el proceso de diseño no es apenas constituido por las fases de proyecto, más por las acciones que se establecen entre ellas. El conjunto de esas relaciones constituyen la organización del proceso.

Si consideramos que dentro del proceso de proyecto hay una secuencia de pasos que parten de una necesidad o un problema hasta la solución en forma de un producto o servicio, se entiende que debería existir en cada paso un conjunto de acciones que deberían ser realizadas por un equipo interdisciplinar formado por individuos con puntos de vista cognitivamente diversos permitiendo una interrelación de múltiples ideas, informaciones, opiniones, teorías en intercambio continuo.

La complejidad da dimensión a la sustentabilidad, exige una postura diferente de diseño, este escrito propone abandonar la definición del diseño como un cuerpo organizado de conocimientos para construir un diseño orgánico que integre disciplinas, es decir, parafraseando a Japiassu (2066) que las instituciones de enseñanza sean lugares de permanentes intercambios y debates permitiendo a los diversos "especialistas" colocar en común sus experiencias.

Como menciona Tronca (2006, p. 76) considerando el mundo en constante transitoriedad, donde el conocimiento aumenta de forma incontrolable y la cantidad de nuevas informaciones disponibles es cada vez mayor, es imposible que persistan maneras reduccionistas de aprendizaje.

\section{Demanda de la Sustentabilidad}

No habrá una verdadera respuesta a la crisis ecológica a no ser en escala planetaria y con la condición de que se opere una auténtica revolución política, social y cultural reorientando los objetivos de la producción de bienes materiales e inmateriales (Guattari, 1990). Las palabras de Félix Guattari, filósofo, psicoanalista y político francés fueron dichas en la década de 90. Antes de él los avisos de la crisis del planeta vinieron de varias fuentes tales como: de la biología con la publicación en 1962 de Rachel Carson en su conocido libro "Primavera Silenciosa". Una década después el libro Small is Beautiful: Economics as if People Mattered fue publicado en 1973 del economista E. F. Schumacher, con un abordaje de los aspectos: económicos, sociales y ecológicos. En el área del diseño Victor Papanek en 1971 publicó el libro "Design for de real world" en que abordaba la responsabilidad social y ambiental del diseñador, el mismo autor publicó en 1995, "Green Imperative” y en 1998 Arquitectura y diseño. 
Se puede percibir que no fue por falta de aviso que los problemas ambientales se intensificaran y nuevamente con varios autores que se manifestaron. En 2003 el libro "Cradle to cradle" del arquitecto William McDonough y del químico Michael Braungart incentivan a pensar diferente, sin residuos; en el libro Colapso: como las sociedades escogen el fracaso o el éxito, el profesor de Geografía de la Universidad de California, Jared Diamond, muestra evidencias de como algunas civilizaciones desaparecieron del mapa por no ser sustentables, ya sea por desperdicio de recursos naturales o aumento de la población, la perdida de florestas o por otras acciones humanas que provocaron la perdida de capacidad de los ecosistemas. En 2006 la publicación "Una verdad inconveniente" de Al Gore presentaba datos y fotos de la situación caótica del planeta. En 2007 el video The story of Stuff de la científica ambiental Annie Leonard fue visto por millones de personas y en 2010 el libro con el mismo título del video muestra datos delos impactos provocados por el hiperconsumo. En 60 años, desde la publicación del libro primavera silenciosa como especie humana hemos hecho muy poco para mudar de rumbo y construir un ambiente sustentable. En el campo del diseño algunos cursos colocaron la disciplina de ecodiseño, diseño y sustentabilidad, gestión ambiental, fueron creados también algunos simposios y congresos de sustentabilidad y desarrollados muchos productos "verdes". En la mayoría de las veces son productos denominados apenas por tener un material "ecológico", "biodegradable" o por usar en su confección material "reciclado".

Goleman (2009) expone que, a pesar del reciclaje de un material, todos los otros impactos continúan existiendo. Eso transforma nuestra noción de "verde" de un juicio aparentemente binario - verde o no verde- en una cuestión más compleja de distinciones, en que cada una de ellas muestra impactos mejores o peores a lo largo de innumerables dimensiones. Cuando el análisis deja de enfocar apenas en los impactos sobre el medio ambiente y analiza también el impacto sobre la salud humana, la matemática es más compleja.

Para McDonough y Braungart (2010) un material por el simple hecho de ser reciclable, no se convierte automáticamente en positivo para el medio ambiente, especialmente si no fue proyectado específicamente para ser reciclado. Adoptar ciegamente aproximaciones ecológicas superficiales sin entender plenamente sus consecuencias puede no ser mejor $-\mathrm{y}$ puede inclusive ser peor- que no hacer nada.

Un análisis más detallado de los impactos ocultos de un producto "verde" o de una acción "ecológicamente correcta" puede revelar apenas una maniobra de mercado que elogian una o dos cualidades positivas de un producto o comportamiento para aumentar su precio en el mercado. Goleman (2009, p. 22) advierte que "considerar un producto verde con base en un único atributo -es ignorar diversos impactos negativos- es mera ilusión”. Un ejemplo es la reutilización de botellas plásticas de agua mineral. Pero la respuesta "ecológicamente correcta" deja de lado un aspecto negativo: el uso de botellas plásticas impone impactos potencialmente negativos para la salud, en función de las sustancias químicas que pasan hacia el agua embotellada.

Goleman (2009) alerta que pasamos por la vida en medio de muchos objetos que compramos, usamos, descartamos, desperdiciamos o guardamos y que cada una de esas cosas tiene una red de impactos que se quedan ocultos a lo largo del camino, de la extracción, producción, transporte, uso y descarte. Destaca que el mundo industrializado está creando un ensopado químico que contamina lentamente el ecosistema y nuestra salud. 
Pero, además del ecosistema y de la salud humana existe la dimensión social, lo que torna el tema de la sustentabilidad aún más compleja. Leonard (2011) expone los problemas sociales de los "minerales de guerra" un término usado para referirse a minerales valiosos cuya venta, tributación o protección abastece conflictos violentos y financia armas, grupos criminales y regímenes inhumanos. El Coltan es un mineral que se encuentra en celulares, tablets, controles remotos, PlayStations. La autora menciona que en el 2000, en el Congo, cuando el kilo refinado de Coltan disparó en el mercado internacional, florestas, parques nacionales, tierras vírgenes y el hábitat de los animales fueron destruidos por la extracción en minas clandestinas y pésimas condiciones de trabajo.

Guattari (1990) mencionaba una articulación llamada ecosofia que sería un abordaje que incluye tres ecologías, la del medio ambiente, la de las relaciones sociales y la de la subjetividad humana. La ecología del medio ambiente es aquella relativa a las transformaciones tecnológicas y económicas de la modernidad relacionadas a los desequilibrios ecológicos que amenazan la vida en el planeta. La ecología de las relaciones sociales dice respecto al deterioro de la calidad de vida. La ecología de la subjetividad humana se relaciona con los comportamientos que dirigen la vida familiar doméstica y la vida de las relaciones como un todo y de los comportamientos empobrecidos.

Existe un paralelo con lo mencionado por Goleman (2009) que debemos conocer tres áreas convergentes.

- La geósfera que incluye el suelo, aire, agua y clima de forma que sean capaces de sustentar la vida del ser humano y de otras especies. El autor menciona que cuando la actividad humana destruye las fuentes naturales como destrucción y desaparición de una floresta tropical, la destrucción de un acuífero, el agotamiento de reservas de florestas, la perdida es, al mismo tiempo, inmediata e irreversible. La deuda de un producto para con la naturaleza puede ser calculada como la suma de los recursos no renovables agotados, más su impacto sobre la naturaleza, como los contaminantes emitidos en la atmósfera, las toxinas lanzadas en el agua o los contaminantes enterrados en aterros sanitarios durante su producción y descarte.

- La biósfera que incluye nuestro organismo, el de otras especies y la vida vegetal. Goleman resalta que los sistemas en el interior de nuestro cuerpo tienen un límite para la acumulación de compuestos extraños, y que dosis mínimas de algunas sustancias pueden tener efectos colaterales jamás previstos. El autor relata que la "toxicidad personificada" calcula cuántas sustancias químicas problemáticas son depositadas en la naturaleza durante el ciclo de vida del producto. Para una cortina de ducha de PVC, tendríamos que calcular el petróleo extraído y procesado, el cloro acrecentado para la producción del polivinil clorado -rico en cancerígenos- del que está hecha la cortina de baño. Así, cuando nos bañamos hay emisiones de gases de ftalatos que se mezclan para dejar la cortina más maleable. Cuando una cortina termina sus días en un basurero, libera lentamente el gas cloro. - La sociósfera está relacionada con preocupaciones humanas como las condiciones de los trabajadores y calidad de vida. Para Goleman el Análisis del Ciclo de Vida tiene negligencia en relación a los impactos sociales. 
La forma de pensar la sustentabilidad debe incluir los tres sistemas en la ecuación. Ya que como mencionaba Guattari las tres ecologías tratan del ambiente entero para dentro y más allá de nosotros, una visión ecológica amplia que considera un ambiente de reciprocidad en que la alteración de un único elemento puede provocar transformaciones para el conjunto, colocando en riesgo el equilibrio del "todo".

Goleman (2009) defiende enfáticamente que precisamos dominar un nuevo tipo de matemática que muestre, con mucha más claridad y un nivel de detalles mayor, las consecuencias de nuestras decisiones, compras en el día a día de forma que podamos clasificar los impactos de los productos y de pensar sobre ellos.

Para este tipo de datos el paradigma necesita de una revolución social, cultural y educativa que reoriente la producción y el consumo de bienes naturales y materiales. Para eso, necesitamos redefinir la palabra sustentabilidad, como equilibrio a lo largo del tiempo, que contemple los ámbitos ambiental, humano y social.

\section{El sueño interdisciplinario}

La forma de pensar el saber fragmentado, mono-disciplinar, tomando como criterio de construcción el punto de vista (el paradigma) de una rama del saber o disciplina auto determinada, con todos sus intereses subyacentes, es responsable del predominio de una inteligencia más bien miope o ciega en la medida en que se sacrifica la capacidad humana normal de reconectar el conocimiento en beneficio de la capacidad de separarse o desconectarse (Japiassu, 2006, p. 15).

La interdisciplinariedad aparece como una nueva actitud hacia el conocimiento. Reconoce las disciplinas, pero promueve, en un ejercicio colectivo del conocimiento (integración posibilitada por la intercomunicación continua). En la concepción de Demo (1997, p. 114) [...] la interdisciplinariedad representa la orquestación intrínseca de las disciplinas, haciendo converger sus diversos puntos de origen. Para Fazenda (1991, p. 31) [...] su principio es siempre el mismo: se caracteriza por la intensidad de las alianzas entre especialistas y la integración de disciplinas.

Según Fazenda (1979, p. 8) "la interdisciplinariedad antes que "una moda" es una relación de reciprocidad, de mutualidad, que presupone una actitud diferente a asumir frente al problema del conocimiento, es decir, es la sustitución de una concepción fragmentaria del ser humano unitario". Para el autor, una condición para la efectividad de la interdisciplinariedad es el desarrollo de la sensibilidad, que requiere entrenamiento en el arte de comprender y esperar, un desarrollo de la creación y la imaginación.

La necesidad de interdisciplinariedad en el campo del diseño es el requisito de la complejidad de la sostenibilidad. Puede ayudar en la comprensión del problema ambiental y en la reforma del pensamiento y la enseñanza del diseño, para integrar disciplinas correspondientes a los problemas que nos desafían. No podemos simplemente contentarnos con la yuxtaposición de disciplinas en vista de la complejidad de los problemas ambientales y sociales. Necesitamos pensar de manera amplia, superar la miopía del conocimiento frag- 
mentado y el punto ciego de los impactos ambientales y sociales de nuestros proyectos, consumos y comportamientos.

Los conocimientos de biología, ecología, química, sociología, psicología entre otros deben implicar transferencias de conocimientos, originando un nuevo cuerpo disciplinar con el diseño, creando un nuevo y mayor patrón epistemológico. Esto, en opinión de quien escribe, es como un sueño, imaginar que el campo del diseño puede ser mejor a la hora de materializar objetos y aportar valores éticos en el comportamiento de la sociedad.

La distancia entre el sueño y la realidad proviene de testimonios de autores que muestran la dificultad de practicar la interdisciplinariedad. Couto (1997) dice que, en el campo del Diseño, la práctica docente ha demostrado que las propuestas efectivas de trabajo interdisciplinario pocas veces se han consolidado en el ámbito universitario. Bomfim (1997) también señaló que cuando un diseñador busca, por ejemplo, utilizar elementos de ergonomía, estética y semiótica en la configuración de un objeto, se enfrenta a situaciones antagónicas, ya que estas ciencias emiten enunciados en diferentes códigos, existiendo ninguna etapa intermedia, algo así como una "ergoestético". Este hecho anecdótico nos hace reflexionar que si no existe una interdisciplinariedad entre el diseño y disciplinas consolidadas en la esquema curricular del curso como son la ergonomía, la estética y la semiótica, ¿Cuál sería el camino hacia la interdisciplinariedad con disciplinas que no forman parte de un curso de diseño, como biología o química?

Follari (1995 y Couto, 1997) mencionan que fuera de la universidad, donde el objetivo no es generar conocimiento, sino resolver problemas prácticos, la duración de la interdisciplinariedad, en general, es más inmediata y se practica con éxito. En estos casos, no hay muchos prejuicios epistemológicos y nadie se preocupa por unir a los distintos profesionales con un objetivo eminentemente práctico.

Además de acercarse a lo real, la apertura al diálogo es considerada por varios autores como una práctica educativa imprescindible. Para Japiassu (2006) "es necesario que todos estén abiertos al diálogo, que sean capaces de reconocer lo que les falta y que pueden o deben recibir de los demás. [...] Para que todos estén abiertos al diálogo, debe haber una conciencia, principalmente individual”. Según Japiassu (2006), la interdisciplinariedad ayuda a cambiar y mejorar. Porque cultiva el deseo de enriquecimiento a través de un nuevo enfoque, el gusto por la combinación de perspectivas y alimenta el deseo de superar los caminos ya recorridos y los conocimientos ya adquiridos.

Le corresponde entonces al educador desarrollar primero un diálogo reflexivo, sólido y crítico que respete las ideas y conocimientos de los demás y que transforme sus conceptos y conocimientos. Además, es necesario fomentar la curiosidad de los estudiantes de manera permanente, para problematizar la situación ambiental, para mostrar la necesidad de conocimientos de otras áreas, con el fin de despertar la búsqueda y desarrollo de nuevos conocimientos fuera y dentro del curso.

\section{Nuevos conocimientos para integrarlos en el diseño}

¿Por qué la armonía, que aparece tan claramente en la naturaleza, no juega un papel esencial en nuestra vida social? Estamos tan fascinados con nuestra omnipotencia, con el po- 
der de inventar y realizar, que quizás hayamos perdido de vista la fuerza de los límites. Pero, ahora nos vemos repentinamente forzados a limitar la explotación de nuestra riqueza material y el aumento de la población [...] debemos aprender nuevamente a salvarnos y moderarnos, encontrando las proporciones adecuadas y efectivas (Blüchel, 2009, p. 51). McDonough y Braungart (2010) mencionan que si queremos prosperar debemos aprender a imitar a la naturaleza en su óptimo sistema eficiente desde la cuna hasta la cuna, en relación a los flujos de nutrientes y al metabolismo, en el que no existe el concepto de desperdicio. Para los autores en un metabolismo biológico, un nutriente es un material o producto diseñado para volver a los ciclos naturales para ser consumido por los microorganismos del suelo u otros animales. También significa abandonar el concepto de la cuna a la tumba, que presupone un depósito para los desechos.

Otro concepto que debería ser reemplazado sería la eco-eficiencia por la eco-eficacia, para McDonough y Braungart (2010, p. 71) "Si la naturaleza se hubiera adherido al modelo humano de eficiencia, habría menos flores en un cerezo y menos nutrientes. Habría menos árboles, menos oxígeno y menos agua pura. Habría menos aves y menos diversidad". De lo anterior, deviene que una disciplina que debe integrarse en el diseño es la biología. Para Blüchel (2009, p. 38)

La investigación sistemática del hombre sobre la competencia de los sistemas biológicos se retrasa. Con eso, se podrían crear miles de nuevos productos, principalmente beneficiosos para la naturaleza, e innumerables problemas de la sociedad, la economía y la industria podrían llevar a soluciones orientadas a la naturaleza.

La biónica como técnica creativa se ha investigado y aplicado superficialmente en el diseño. La bibliografía y producción con el uso de la técnica han estado en función de innovar y optimizar formas y funciones de productos basados en sistemas naturales. Sin embargo, el enfoque ecológico no se ha incorporado a la biónica. Aquí aparece un campo de investigación que integra la biología bajo una mirada de complejidad, inteligencia ecológica y conciencia de la interrelación de la naturaleza.

Otra interesante integración de la biología con varias áreas es la biomimética, que significa imitación de la vida. Para Benyus (1997), es una nueva ciencia que estudia los modelos de la naturaleza y luego se inspira en ellos o los imita, con el objetivo de resolver problemas humanos. El biomimetismo utiliza un estándar ecológico para juzgar la corrección de las innovaciones, determinando qué funciona, qué es apropiado y qué dura. Se presenta como un término más amplio que nos permitirá mirar la naturaleza de una manera diferente. Precisamente porque esta disciplina apareció en la frontera de disciplinas como la agricultura, la ecología, la medicina, la ingeniería de materiales, la tecnología de la información. Es el resultado de una acción interdisciplinar que puede contribuir a la creación de una nueva disciplina con el lenguaje y la necesidad del diseño.

Ewel (1991 y Benyus, 1997) recomiendan, "imita la estructura vegetal de un ecosistema y obtendrás la funcionalidad que buscas". McDonough y Braungart (2010) ejemplifican cómo esta imitación puede ser eficiente en el diseño. Para los autores, todos los materiales 
de las hormigas, incluidas las armas químicas más letales, son biodegradables y, una vez devueltos a la tierra, se convierten en nutrientes.

Además de la biología y la ecología, una disciplina que también debe integrarse en el diseño es la química. La información que tienen los diseñadores sobre los materiales se centra mayoritariamente en aspectos estéticos, de precio y de resistencias, los aspectos ambientales son minimizados o superficiales. El diseñador no tiene suficiente información sobre los datos de toxicidad, los resultados de las pruebas de materiales y los resultados del análisis del ciclo de vida. Goleman (2009) recuerda que las toxinas flotan en el aire, el agua y el suelo, o provienen de una larga lista de objetos que van desde pinturas hasta alfombras, computadoras, teléfonos celulares y muebles.

Por otro lado, la idea de sostenibilidad no se limita a los materiales, al contrario, empieza por ellos. El material reciclado requiere una tarea de recuperación y una tarea de transformación que implica consumo de energía y generación de residuos. Una lata de aluminio se compone de una combinación de aluminio y manganeso y un porcentaje de magnesio. En un proceso de reciclaje, los materiales se fusionan para producir un metal más débil y, por lo tanto, menos útil. Sólo el "reciclado" de la biósfera puede devolver el material consumido a su estado inicial de recurso natural.

McDonough y Braungart (2010) destacan que un traje de poliéster y una botella de agua son dos ejemplos de productos que ofrecen a los consumidores mas aditivos que no han solicitado y que pueden ser perjudiciales para su salud. Los autores continúan mencionando que a lo largo de la investigación encontraron que varios productos como: un mouse de computadora, una afeitadora, un secador de pelo, un videojuego y un reproductor de CD durante su uso emitían compuestos teratogénicos y cancerígenos. Los autores mencionan que otra sustancia peligrosa en los plásticos son los ftalatos que pueden penetrar en el cuerpo por contacto, como es el caso de los flotadores en piscinas en contacto con la piel fina de los niños.

Con lo dicho a lo largo del texto, se necesita una mayor integración de disciplinas con conocimientos específicos de impacto en la salud y el medio ambiente, así como disciplinas en las que aprehender nuevas formas de configuración de objetos.

\section{Campo y naturaleza del diseño}

Definir los límites del campo del diseño es una tarea compleja, por no decir difícil, ya que el diseño se caracteriza por ser una actividad multidisciplinar en la que existe el trabajo simultáneo de un abanico de disciplinas y conocimientos diversos. Para Bomfim (1997), lo que parece original en la relación entre teoría y práctica en el diseño, es el hecho de que el conocimiento que demanda la praxis pertenece a diferentes ramas de las ciencias clásicas. De esta forma, el campo del diseño sería multidisciplinar y, por tanto, con límites flexibles entre las distintas áreas del conocimiento, de tal forma que no se puede trabajar la actividad del diseño y la investigación en diseño sin recurrir a autores de diferentes disciplinas. El diseño, desde su surgimiento como disciplina, necesitaba alianzas entre varias áreas: ergonomía, psicología, matemáticas, sociología, etc. En este contexto la interacción ha sido a nivel multidisciplinar, integrando disciplinas o contenidos. 
En la búsqueda de una definición sobre el campo del diseño, Monat, De Campos y Lima (2008) defienden la tesis de que el diseño actúa como un metaconocimiento, al igual que la Filosofía, a pesar de ser un conocimiento distinto. Dentro de lo multidisciplinar en la que se establece su campo de actuación, destacan que la actividad del diseño consiste en traducir e interpretar conocimientos de otras áreas en objetos adaptados al hombre, como usuario, a través de métodos de trabajo específicos. Es la realización del conocimiento en objetos tangibles que pueden ser utilizados por los seres humanos. En este sentido, los autores destacan que el diseño tiene una estructura epistemológica comparable a la de la filosofía, ya que la naturaleza de su conocimiento también se basa en la relación con otros conocimientos y en cómo se configuran posteriormente para fines específicos.

El objeto concreto resultante del proceso de diseño, supone la existencia de un sujeto y un contexto, ya que como disciplina de proyecto el diseño se basa en el uso social del objeto, que engloba su interpretación objetiva y subjetiva, la conexión con experiencias pasadas, la relación con el lenguaje y repertorio de la asignatura y los impactos causados por el resultado del proceso de diseño en el medio ambiente.

Las interpretaciones teóricas del diseñador y el tema son complejas. Si el diseñador no se apoya en la teoría de varias disciplinas: sociología, biología, economía, ética, ecología, etc., se vuelve difícil lograr un objeto apto para uso social y ambiental.

\section{Sostenibilidad y Diseño}

Hace unas décadas, la idea de un mundo finito no se consideraba una limitación, ya que se creía que no había límites: el razonamiento de FW Taylor (y Deforge, 1994) era que el infinito de la producción se alimentaría del infinito de recursos y tirado por el infinito del consumo. Hoy se comprueba que el alto desarrollo tecnológico, el aumento del consumo y la búsqueda del alto estándar de confort, crearon, por otro lado, la degradación del medio ambiente y la salud humana.

Los diferentes enfoques, técnicas y herramientas de diseño centradas en la sostenibilidad (ecodiseño, diseño ecológico, diseño para el medio ambiente) también cobraron fuerza desde los años 90.

Thackara también apunta al diseño y sus alternativas para un mundo complejo. El autor señala que actualmente muchos diseñadores ya están diseñando servicios y sistemas visiblemente menos dañinos para el medio ambiente y más socialmente responsables, pero enfatiza la necesidad de cambios, proponiendo un cambio de paradigma: "En esta nueva era de innovación colaborativa, los diseñadores deberán tener que evolucionar de autores individuales de objetos a facilitadores del cambio entre grandes grupos de personas" (Thackara, 2008, p. 21).

Así, se observa que el diseño para la sustentabilidad debe realizarse de manera colaborativa y debe buscar trabajar bajo los tres niveles de desarrollo sustentable: ser económico, no dañino para el medio ambiente y accesible para todos. Como ya se mencionó, ya se ha avanzado mucho en este aspecto, pero es necesario considerar que aún vivimos bajo una economía de política de consumo, en un mundo con enormes desigualdades sociales. Con 
esto, el poder que el diseño tiene sobre las personas puede considerarse a la vez sorprendente y astuto, convirtiéndonos en cómplices de este consumismo acentuado.

$\mathrm{Al}$ respecto, Batista (2007) defiende la tesis de que las características de los objetos y soluciones desarrolladas por el diseño sufren imposiciones socioeconómicas y culturales, ya que son inseparables de las líneas de expansión económica. Así, su argumento es que existe una 'racionalidad implícita' en los proyectos de diseño y los objetivos de su producción no promueven la equidad, generando desigualdad debido a la dinámica del mercado, ya que solo quienes tienen poder adquisitivo pueden adquirir un bien, por indispensable que sea. El autor plantea varias disparidades a partir del tema de la sostenibilidad y su relación con el diseño, justificando su pensamiento en el hecho de que los proyectos de diseño deben tener una mirada sistémica que señale el alcance, la complejidad y la diversidad de factores que intervienen en su realización. En este sentido, es importante considerar que los desarrollos no pueden estar limitados por requisitos y parámetros técnicos, y es necesario considerar las diferentes dimensiones de la vida social y el contexto en el que se insertarán. Autores como Deforge y Papanek, abogan por una amplia formación y práctica profesional comprometida con los valores éticos. Si este no es el caso, la sostenibilidad jugará un papel desconcertante, como "ideología sin un sustrato práctico". Batista (2007) también señala:

Observamos que la incorporación acrítica de los supuestos de sustentabilidad en el campo del Diseño Industrial, puede establecer un proceso de auto-legitimación de productos éticamente cuestionables y contribuye a proyectos de productos que se desvanecen técnica y culturalmente. Argumentamos el discurso de la sostenibilidad como un dispositivo utilizado para mitigar el impacto de las estrategias competitivas de la economía capitalista, centradas en la destrucción de los recursos naturales y la emulación del consumo. Este discurso intenta mitigar los efectos de la degradación social y ambiental, sugiriendo que el capitalismo se puede racionalizar, humanizar y promover la eliminación de las desigualdades sociales.

Es un hecho que las preocupaciones por el desarrollo socioeconómico y los impactos generados deben ser constantes, ya que sus consecuencias comprometen el presente y el futuro de la humanidad. Como señala Batista (2007), "el debate sobre la sostenibilidad nos lleva a una visión más integral del mundo en el que vivimos y del sistema económico al que estamos sometidos". Por lo tanto, el enfoque de la sostenibilidad debe ser considerado bajo un pensamiento epistemológico integral, analizado de manera crítica y efectiva y no traducido en "intervenciones apologéticas e ingenuas".

Parece que una disciplina interdisciplinar del diseño tendría que reconocer que los demás conocimientos que componen el plan de estudios actual de los cursos de diseño no satisfacen la demanda de diseño responsable. Todavía hay un gran desconocimiento de leyes, métodos e ideas de diferentes disciplinas para ayudar en el proyecto. Leonard (2011, p. 120) destaca este problema: 
La etapa de diseño establece: los componentes que necesitan ser extraídos o creados; la cantidad de energía gastada en la fabricación y uso del producto; la presencia o ausencia de sustancias tóxicas; la vida útil del producto; la facilidad o dificultad de reparación; su capacidad de reciclaje; Daños causados por enterrar o quemar el producto, si no es reciclable.

Según McDonough y Braungart (2005), la etapa de diseño es el "primer signo de la intención humana” y solo puede conducir a mejoras, como eliminar el peso y el impacto ambiental, o conducir realmente a una reevaluación de los paradigmas actuales. Para los autores, ni la salud de los sistemas naturales ni la comprensión de su complejidad e interrelación se han considerado en el diseño. Los autores también señalan que muchas veces la intención del diseño es solo crear un producto atractivo que sea asequible, que cumpla con las regulaciones, que tenga un desempeño aceptable y que dure lo suficiente para satisfacer las necesidades de los mercados. Sin embargo, señalan que, si bien los productos satisfacen los deseos de fabricantes y consumidores, no están diseñados para beneficiar la salud humana y ecológica.

En este sentido, se observa que la discusión que aquí se propone es oportuna y señala el gran desafío para el campo del Diseño en lo que respecta al desarrollo de productos, servicios y sistemas sostenibles.

\section{Disciplina interdisciplinaria para el diseño sostenible}

Si bien la división del conocimiento en ciencias y disciplinas ha sido la forma de organizar la transmisión del conocimiento de una manera más didáctica, esto ha fragmentado el campo del diseño en titulaciones y especializaciones que buscan una identidad e independencia. Las propuestas para compensar la especialización disciplinar y solucionar los problemas del desconocimiento del diálogo se han denominado multidisciplinares, pluridisciplinares e interdisciplinares.

Las disciplinas se proponen simultáneamente sin expresar explícitamente las relaciones que puedan existir entre ellas. También se presenta como multidisciplinar, con la existencia de relaciones complementarias entre disciplinas más o menos similares. Este es el caso de las contribuciones mutuas de diferentes enfoques de diseño (diseño sostenible, gestión del diseño, diseño emocional, etc.).

Según Sommerman (2008, p. 29) "La pluridisciplinariedad es la yuxtaposición de disciplinas más o menos similares, dentro de un mismo sector del conocimiento”. Por ejemplo, ecología y diseño; administración y diseño; psicología y diseño. Es una yuxtaposición que tiene como objetivo mejorar u orientar las relaciones entre estas disciplinas. Para Sommerman (2008), esto resulta ser una relación de mero intercambio de información, una simple acumulación de conocimientos, sin aporte a una modificación profunda de la base teórica, problemática y metodológica de estas ciencias en su individualidad; es una comunicación que no cambia internamente ya que no existe una interacción profunda.

La interdisciplinariedad, por otro lado, se ocupa de la interacción de dos o más disciplinas. Estas relaciones pueden implicar transferencias de leyes, métodos, conocimientos de 
una disciplina a otra, creando en algunos casos un nuevo cuerpo disciplinario como, por ejemplo, el biomimetismo.

Para Japiassú (1991), la interdisciplinariedad es un método de investigación y enseñanza capaz de hacer que dos o más disciplinas interactúen entre sí. Esta interacción puede ir desde la simple comunicación de ideas hasta la integración mutua de conceptos en terminología, metodología, procedimientos, datos y organización de la investigación.

Lo que caracteriza a la interdisciplinariedad, según Sommerman (2008), es la intención de establecer vínculos entre disciplinas para lograr un conocimiento más integral, al mismo tiempo diversificado y unificado. Los auténticos estudios interdisciplinares suponen una investigación común y un diálogo o debate a través del cual se consolidaría un nuevo conocimiento.

Es común ver autores que tratan el diseño como una actividad interdisciplinar. Sin embargo, para (Couto, 1997), la falsa imagen de que se está dando un trabajo interdisciplinario en la docencia surge de ideas equivocadas, por ejemplo, que un docente en un curso, cuando enseña en otro curso, está estableciendo una relación interdisciplinar.

De lo anterior se desprende que el concepto de interdisciplinariedad es más complejo que el de multidisciplinar y pluridisciplinar que predomina en la enseñanza del diseño. La Tabla 1 destaca algunos enfoques y disciplinas que pueden formar parte de una disciplina interdisciplinaria a favor de la sostenibilidad:

\begin{tabular}{|l|l|}
\hline Enfoques & Disciplinas \\
\hline Comportamiento de los individuos & Sociología, Psicología, Antropología \\
\hline Impactos ambientales y sociales & $\begin{array}{l}\text { Química verde, Ecología industrial, Medicina, } \\
\text { Biomimética, Sociología, Ética }\end{array}$ \\
\hline Impacto global & $\begin{array}{l}\text { Ecología, Sociología, Filosofía, Economía } \\
\text { verde, Marketing, Logística }\end{array}$ \\
\hline $\begin{array}{l}\text { Interacciones entre el ser humano y el medio } \\
\text { ambiente }\end{array}$ & $\begin{array}{l}\text { Psicología, Ecología, Ética, Economía verde, } \\
\text { Sociología }\end{array}$ \\
\hline Interacciones objeto-ambiente & $\begin{array}{l}\text { Biología, Ecología, Ecología industrial, Química } \\
\text { verde, Biomimética }\end{array}$ \\
\hline Enfoque y disciplinas para una nueva disciplina interdisciplinar \\
\hline $\begin{array}{l}\text { Interacción humano-objeto-contexto (geósfera, } \\
\text { biósfera y sociósfera) }\end{array}$ & $\begin{array}{l}\text { Antropología, Biología, Biomimética, Ecología, } \\
\text { Ecología Industrial, Economía Verde, Ética, } \\
\text { Filosofía, Logística, Marketing, Medicina, } \\
\text { Química Verde, Psicología, Sociología }\end{array}$ \\
\hline
\end{tabular}

Tabla 1. Enfoques y disciplinas.

Para una nueva disciplina interdisciplinaria, la transferencia de conceptos debe realizarse a través de la investigación, el diálogo y los debates de especialistas en cada área para comprender la relación entre la problemática ambiental, la sustentabilidad y el diseño. 
Observando la constitución de estos dos campos y los criterios presentados en la Tabla 1, existe la posibilidad de ir en la dirección de la constitución de una nueva disciplina interdisciplinar para el diseño sustentable.

Esta nueva disciplina debe permitir al estudiante de diseño asumir cada día más contenidos de conocimiento en diferentes áreas, permitiéndole realizar acciones éticas e intencionales para un cambio de paradigma y a favor de la salud humana y ambiental.

Para aplicar la visión interdisciplinaria es necesario presentar primero la disciplina. Así, utilizamos los criterios de Fazenda (1979, p. 29), que presenta siete criterios citados por Heinz Heckhausen para caracterizar la naturaleza de una disciplina dada y distinguirla de las demás. La Tabla 2 muestra un intento de delimitar una disciplina interdisciplinar a favor de la sostenibilidad.

\begin{tabular}{|l|l|}
\hline $\begin{array}{l}\text { 7 criterios para caracterizar la naturaleza } \\
\text { de una disciplina dada }\end{array}$ & $\begin{array}{l}\text { Características de una disciplina } \\
\text { interdisciplinaria }\end{array}$ \\
\hline $\begin{array}{l}\text { 1. Dominio material: comprendería la serie de } \\
\text { objetos que trata una disciplina }\end{array}$ & $\begin{array}{l}\text { Debe abordar la comprensión del } \\
\text { comportamiento humano y la complejidad } \\
\text { del equilibrio ambiental }\end{array}$ \\
\hline $\begin{array}{l}\text { 2. Dominio de estudio: definido por el ángulo } \\
\text { específico desde el cual la disciplina delimita } \\
\text { su dominio material }\end{array}$ & $\begin{array}{l}\text { Relación de la vida humana y el medio } \\
\text { ambiente }\end{array}$ \\
\hline $\begin{array}{l}\text { 3. Niveles de integración teórica de los } \\
\text { conceptos fundamentales y unificadores de } \\
\text { una disciplina }\end{array}$ & $\begin{array}{l}\text { Interrelación de conocimientos y métodos } \\
\text { dentro de un enfoque interdisciplinario a } \\
\text { través de debates, diálogos e investigaciones }\end{array}$ \\
\hline $\begin{array}{l}\text { 4. Métodos propios para aprehender y } \\
\text { transformar fenómenos }\end{array}$ & $\begin{array}{l}\text { Métodos de diferentes disciplinas: } \\
\text { Antropología, Biología, Biomimética, Ecología, } \\
\text { Ecología Industrial, Economía Verde, Ética, } \\
\text { Filosofía, Logística, Marketing, Medicina, } \\
\text { Química Verde, Psicología, Sociología }\end{array}$ \\
\hline $\begin{array}{l}\text { 5. Herramientas de análisis que tienen en } \\
\text { cuenta la estrategia lógica, el razonamiento } \\
\text { matemático y la construcción de modelos }\end{array}$ & $\begin{array}{l}\text { Métodos de diferentes disciplinas: } \\
\text { Antropología, Biología, Biomimética, Ecología, } \\
\text { Ecología Industrial, Economía Verde, Ética, } \\
\text { Filosofía, Logística, Marketing, Medicina, } \\
\text { Química Verde, Psicología, Sociología }\end{array}$ \\
\hline 6. Aplicaciones & $\begin{array}{l}\text { Diseño de productos (objetos), servicios y } \\
\text { proyectos visuales (señalización e información) }\end{array}$ \\
\hline Contingencias históricas & $\begin{array}{l}\text { Integración de las contingencias históricas de } \\
\text { cada disciplina }\end{array}$ \\
\hline
\end{tabular}

Tabla 2. Características de la disciplina interdisciplinaria. 
Observando la constitución de estos dos campos y los criterios presentados anteriormente, existe la posibilidad de ir en la dirección de la constitución de una nueva disciplina interdisciplinar para el diseño sostenible.

Una disciplina interdisciplinaria para la sustentabilidad debe generar nuevos conceptos, conocimientos y métodos para reducir el consumo, hacer más felices y saludables a las personas, comprender los impactos ambientales como resultado de las acciones humanas y hacer transparentes los impactos a lo largo del ciclo de vida de los productos. También se debe evitar el desarrollo de productos "verdes" que carecen de características de bajo impacto y requieren "ecomarketing" debido al desconocimiento de los diseñadores sobre los impactos de las materias primas o procesos que causan impactos ambientales, sociales y de salud. La disciplina puede contribuir a provocar la reflexión y, a través de la innovación, encontrar soluciones para un diseño sostenible. La Tabla 3 a continuación muestra el programa de esta nueva disciplina para los cursos de pregrado:

\section{Disciplina}

Fundamentos interdisciplinarios de la sostenibilidad para el diseño sostenible $72 \mathrm{~h}$ (4 créditos)

\section{Tema}

La sostenibilidad como entidad compleja basada en un concepto interdisciplinario. Estudios de las relaciones de factores económicos, sociales y ecológicos en el desarrollo de productos (gráficos o industriales) y servicios

\section{Objetivos}

1. Comprender la complejidad de la sostenibilidad

2. Comprender el problema ambiental

3. Conocer la trayectoria cronológica y multidimensional del nuevo paradigma ambiental

4. Conocer las propuestas de nuevos escenarios sostenibles

5. Conocer los métodos de diferentes disciplinas a favor de la sostenibilidad

6. Promover una reflexión sobre las implicaciones del diseño ante estos nuevos escenarios sostenibles

\section{Enseñanza - Estrategias de aprendizaje}

Estudio de textos básicos y complementarios de diferentes disciplinas

Investigaciones y debates

Seminarios con expertos

Educación cinematográfica

Producción de textos científicos

\section{Bibliografía básica}

Goleman, $\boldsymbol{D}$. Inteligencia ecológica: El impacto de lo que consumimos y los cambios que pueden mejorar el planeta. Elsevier, Río de Janeiro, 2009.

Leonard, $\boldsymbol{A}$. La historia de las cosas: de la naturaleza a la basura, qué pasa con todo lo que consumimos. Río de Janeiro: Zahar, 2011. 
Manzini, E. Diseño para la innovación social y la sostenibilidad. Rio de Janeiro. documentos electrónicos, 2008.

Manzini, E., Vezzoli, C. El desarrollo de productos sostenibles: los requisitos medioambientales de los productos industriales. Editor de la Universidad de São Paulo EDUSP, São Paulo, 2002.

McDonough, W., Braungart, $M$. Cradle to Cradle (De cuna a cuna) rediseñando la forma en que cortamos las cosas. Mc GrawHill. Madrid, 2005.

\section{Bibliografía complementaria}

Benyus, J. M., Biomimética: innovación inspirada en la naturaleza. São Paulo: Cultrix, 1997.

Bluchel, K. G. Bionics: ¿cómo podemos utilizar la ingeniería a nuestro favor? São Paulo: Pub. House Lobmaier, 2009.

Guattari, F. Las tres ecologías. Campinas. Papirus, 1990.

Kazazian, T. (org.). Habrá una era de cosas ligeras: diseño y desarrollo sostenible. São Paulo: Editora Senac São Paulo, 2005.

Laguna; Pádua, J.A. Qué es la ecología. San Pablo. Abril Cultura / Brasiliense, 1985.

Papapnek, V. Diseño para el mundo real; ecología humana y cambio social. Nueva York, Pantheon Books, una división de Random House, 1971.

Papanek, V. Arquitectura y diseño: ecología y ética, Ediciones 70, Lisboa 1995.

Thackara, J. Plano B: diseño y alternativas viables en un mundo complejo. São Paulo: Saraiva: Versar, 2008.

Tabla 3. Programa de una disciplina interdisciplinaria.

Una disciplina interdisciplinar que proporcione nuevos marcos teóricos puede ayudar a afrontar la complejidad de la sostenibilidad y dar cuenta de problemas relacionados con cuestiones ambientales, ecológicas, éticas, sociales y culturales que impregnan la actividad del diseño.

\section{Conclusión}

El artículo buscó compartir reflexiones e ideas sobre la necesidad de una interdisciplinariedad como demanda de sostenibilidad. La postura interdisciplinar no solo ayudaría a comprender la complejidad del tema, sino que facilitaría la integración de nuevas disciplinas, el trazado de nuevos caminos y la creación de nuevas investigaciones. Puede ayudar en la comprensión del problema ambiental y en la reforma del pensamiento y la enseñanza del diseño, para integrar disciplinas correspondientes a los problemas que nos desafían. 
También buscamos revisar el concepto de "verde", que se ve erróneamente como un estatus, eslogan o adjetivo para vender más y atraer consumidores, para empezar a ver y proyectar lo "verde" como un proceso complejo de muchas dimensiones.

El diseño para la sostenibilidad no debe verse como una propuesta audaz, que resuelve los problemas ambientales que asolan el planeta, ni será efectivo si lo practican solo los diseñadores. Se debe considerar un enfoque complejo, que requiere del conocimiento de varias disciplinas que permitan su inserción en el proceso de desarrollo del producto, repensando los conceptos productivos, el sistema político y económico en el que estamos insertos, así como nuestra postura como profesionales, usuarios y consumidores.

\section{Notas}

1. Complejo puede entenderse como un tejido de eventos, acciones, interacciones, determinaciones, azar que constituye el proceso.

\section{Referencias}

Batista, W. B. (2007). Desenho Industrial e a ideologia da sustentabilidade. In: $4^{\circ}$ Congresso Internacional de Pesquisa em Design, Rio de Janeiro.

Benyus, J. M. (1997). Biomimética - Inovação inspirada na natureza. São Paulo: Cultrix.

Bluchel, K. G. (2009). Biônica: como podemos usar a engenharia a nosso favor. São Paulo: Pub. House Lobmaier.

Bomfim, G. A. (1997). Fundamentos de uma Teoria Transdisciplinar do Design: Morfologia dos objetos de uso e sistemas de comunicação. In: Estudos em Design, volume V, número 2, Rio de Janeiro.

Couto, R. M. de S. (1997). Movimento interdisciplinar de designers brasileiros em busca de educação avançada. Tese de doutorado. PUC-Rio.

Deforge, Y. (1994). Por um Design Ideológico. In: Estudos em Design, volume II, número 1, Rio de Janeiro.

Demo, P. (1997). Conhecimento moderno: Sobre ética e intervenção do conhecimento. Petrópolis: Vozes.

Fazenda, I. (1991). Interdisciplinaridade um projeto em parceria. Edições Loyola. São Paulo.

Goleman, D. (2009). Inteligência ecológica: O impacto do que consumimos e as mudanças que podem melhorar o planeta. Elsevier, Rio de Janeiro.

Guattari, F. (1990). As três ecologias. Campinas: Papirus.

Japiassu, H. (2006). O sonho transdisciplinar: e as razões da filosofia. Rio de Janeiro: Imago.

Leonard, A. (2011). A história das coisas: da natureza ao lixo, o que acontece com tudo que consumimos. Rio de Janeiro: Zahar.

McDonough, W. \& Braungart, M. (2005). Cradle to Cradle (De la cuna a la cuna) rediseñando la forma em que hacemos las cosas. Madrid: Mc GrawHill. 
Monat, A.; De Campos, J. L. \& Lima, R. C. (2009). Metaconhecimento: Um esboço para o design e seu conhecimento próprio. In: Biblioteca On-line de Ciências da Comunicação, 2008. Disponível em http://www.bocc.uff.br/_listas/tematica.php?codtema=33. Acesso em 05 de dez.

Papanek, V. (1977). Design para el mundo real: Ecología humana e cambio social. Madrid: Ediciones Blume.

Sommerman, A. (2008). Inter ou Transdisciplinaridade? Da fragmentação do discurso ao novo diálogo entre saberes. São Paulo: Paulus.

Thackara, J. (2008). Plano B: o design e as alternativas viáveis em um mundo complexo. São Paulo: Saraiva: Versar.

Tronca, D. S. (2006). Transdisciplinaridade em Edgar Morin. Caxias do Sul. RS.

\begin{abstract}
This writing presents a reflection on the need for interdisciplinary practice in the field of design. Through a documentary research, the complexity of sustainability in the environmental, human, social, cultural and technological fields is presented, showing the importance of interdisciplinarity as a means of integration with disciplines that are not part of the curricular plan of design courses. The work emphasizes the need to integrate disciplines such as biology, ecology, chemistry, environmental engineering, sociology, anthropology with the field of product design through critical and solid dialogue promoted by a curriculum that places these disciplines as elective or compulsory.
\end{abstract}

Keywords: Interdisciplinarity - product design - sustainability - design teaching - discipline

Resumo: Este texto apresenta uma reflexão sobre a necessidade da prática interdisciplinar no campo do design. Por meio de uma pesquisa documental, é apresentada a complexidade da sustentabilidade nos campos ambiental, humano, social, cultural e tecnológico, mostrando a importância da interdisciplinaridade como meio de integração com disciplinas que não fazem parte do plano curricular dos cursos de design. O trabalho enfatiza a necessidade de integrar disciplinas como biologia, ecologia, química, engenharia ambiental, sociologia, antropologia com o campo do design de produto por meio de um diálogo crítico e sólido promovido por um currículo que coloque essas disciplinas como eletivas ou obrigatórias.

Palavras chave: Interdisciplinaridade - design de produto - sustentabilidade - ensino de design - disciplina 